attempt to explain phenomena. But what if the phenomenon we are trying to explain does not fit this model? Complexity science argues that when we try to model a nonlinear dynamic system with traditional statistical models, we can never begin to understand spontaneous, self-organizing systems like those in health care $(\mathrm{McDaniel}$ and Driebe 2001). This may help explain why we find low r-squares in explanatory models, why the speed of innovation varies in different organizations, and why similar interventions in different settings may have different "uptakes."

Robert G. Badgett, M.D. Mary Jo V. Pugh, Ph.D., R.N. Veterans Evidence-based Research, Dissemination, and Implementation

Center San Antonio, Texas Badgett@uthscsa.edu

\title{
References
}

Greenhalgh, T., R. Glenn, F. Macfarlane, P. Bate, and O. Kyriakidou. 2004. Diffusion of Innovations in Service Organizations: Systematic Review and Recommendations. Milbank Quarterly 82(4):581-629.

McDaniel, R.R., and D.J. Driebe. 2001. Complexity Science and Health Care Management. Advances in Healthcare Management 2:11-36.

Plsek, P.E. 2001. Appendix B: Redesigning Health Care with Insights from the Science of Complex Adaptive Systems. In Crossing the Quality Chasm: A New Health System for the 21st Century, edited by Institute of Medicine, 309-22. Washington, D.C.: National Academy Press.

Plsek, P.E., and T. Greenhalgh. 2001. Complexity Science: The Challenge of Complexity in Health Care. BMJ 323:625-8.

\section{Author's Response}

I agree with Drs. Badgett and Pugh that complexity science is an excellent explanatory model for analyzing the spread of innovation in service organizations, for precisely the reasons they have outlined. Table 1 of our article lists "complexity studies" as one of 13 research traditions that contributed to our systematic review. But the other 12 traditions provided additional insights that enriched our overall model. Depending 
on the example being considered, any one (or more) of these traditions might provide a useful "lens" through which to analyze a situation, explain empirical findings, consider ways forward, and estimate the likely success of interventions. The "lens" of complex systems is, of course, particularly helpful when the example being considered lies to the far left of our Figure 2. It is less fit for this purpose in other (perhaps less common) circumstances.

The implied suggestion that there is a "best model" for studying the diffusion of innovations in organizations raises the important methodological question of whether we were right to take a descriptive rather than a normative perspective in our review. In this challenging piece of work, we deliberately redefined the reviewer's task as "summarizing how different groups of scientists in the past have conceptualized and researched the problem" rather than "defining how the problem should bave been conceptualized and researched and summarizing the studies that have met these criteria." The normative approach (as taken by the Cochrane and Campbell reviews) typically requires reviewers to reject more than 90 percent (and, not uncommonly, more than 99\%) of the empirical literature as either irrelevant or methodologically flawed. This makes such reviews hard to follow and not very useful (Loke and Derry 2003). As we justify in more detail in our forthcoming article on methods, we anticipate that a systematic descriptive approach will lead reviewers to work more closely with policymakers, ask better research questions, evaluate primary studies more rigorously, and produce more illuminative summaries (Greenhalgh et al. 2005).

But the proof of the pudding will be in the eating. We invite other teams to contact us if they plan to use meta-narrative review in a secondary research study, so that methodological questions not yet answered can be addressed prospectively.

Trisha Greenhalgh, M.D.

University College London p.greenhalgh@pcps.ucl.ac.uk

\section{References}

Greenhalgh, T., G. Robert, F. Macfarlane, P. Bate, O. Kyriakidou, and R. Peacock. 2005. Storylines of Research: A Meta-Narrative Perspective on Systematic Review. Social Science and Medicine. In press.

Loke, Y., and S. Derry. 2003. Does Anybody Read "Evidence-Based" Articles? BioMed Central Medical Research Methodology 3:14. 